\title{
Molecular mechanism underlying Pyropia haitanensis PhHsp22-mediated increase in the high-temperature tolerance of Chlamydomonas reinhardtii
}

\author{
Jing Chang ${ }^{1,2,3} \cdot$ Jianzhi Shi ${ }^{1,2,3} \cdot$ Jianzhang Lin ${ }^{1,2,3} \cdot$ Dehua $\mathrm{Ji}^{1,2,3} \cdot$ Yan Xu $\mathrm{X}^{1,2,3} \cdot$ Changsheng Chen $^{1,2,3}$. \\ Wenlei Wang ${ }^{1,2,3} \cdot$ Chaotian Xie ${ }^{1,2,3}$ (D)
}

Received: 22 August 2020 / Revised and accepted: 7 December 2020 / Published online: 26 January 2021

(C) The Author(s) 2021

\begin{abstract}
Global warming is one of the key limiting factors affecting the cultivation of Pyropia haitanensis which is an economically important macroalgae species grown in southern China. However, the mechanism underlying the high-temperature tolerance of $P$. haitanensis remains largely unknown. In a previous study, we showed that the expression of the small heat shock protein 22 gene (Hsp22) is upregulated in P. haitanensis in response to high-temperature stress, but the associated regulatory mechanism was not fully elucidated. In this study, a transgenic Chlamydomonas reinhardtii expression system was used to functionally characterize P. haitanensis Hsp22. Our analyses indicated that the C-terminal of PhHsp 22 is highly conserved and contains an Acrystal structure domain. A phylogenetic analysis revealed PhHsp22 is not closely related to small heat shock protein genes in other species. Additionally, PhHsp 22 expression significantly increased at 3 and $6 \mathrm{~h}$ after initiating $33^{\circ} \mathrm{C}$ treatment, which improved the survival rate of transgenic $C$. reinhardtii during the early stage of high-temperature treatment. The further transcriptome analysis revealed that PhHsp22 expression can promote pathways related to energy metabolism, metabolites metabolism, and protein homeostasis in transgenic $C$. reinhardtii cells exposed to high temperatures. Therefore, PhHsp22 may be crucial for the response of Pyropia species to high-temperature stress. Furthermore, this gene may be useful for breeding new high-temperature algal strains.
\end{abstract}

Keywords PhHsp22 $\cdot$ Pyropia haitanensis $\cdot$ Chlamydomonas $\cdot$ Rhodophyta $\cdot$ High-temperature stress $\cdot$ Homeostasis

\section{Introduction}

Pyropia species are economically important marine red algae used to produce food, fertilizer, and medicine (Blouin et al. 2011; Brawley et al. 2017; Cao et al. 2020). With the expansion of artificial seeding and the development of a floating culture method, the farming and processing of Pyropia species

Jing Chang and Jianzhi Shi contributed equally to this work.

Wenlei Wang

wlwang@jmu.edu.cn

Chaotian Xie

ctxie@jmu.edu.cn

1 Fisheries College, Jimei University, Xiamen 361021, China

2 Fujian Engineering Research Center of Aquatic Breeding and Healthy Aquaculture, Xiamen 361021, China

3 Key Laboratory of Healthy Mariculture for the East China Sea, Ministry of Agriculture, Xiamen 361021, China is now a major part of the largest seaweed industries in East Asian countries, including China, Japan, and South Korea (Sahoo et al. 2002; Hwang et al. 2019). However, because of global warming, seawater temperatures have gradually increased in recent years which has adversely affected the cultivation and yield of Pyropia species (Shi et al. 2017; Wang et al. 2018a; Hwang et al. 2019). To adapt to global climate change and to continue to develop improved methods for cultivating Pyropia species, several high-temperature (HT) tolerant Pyropia haitanensis strains (Z-26, Z-61, and ZS-1) have been selected and are now widely cultivated in southern China (Chen et al. 2008). Although the mechanisms underlying this high-temperature tolerance have been investigated (Shi et al. 2017; Wang et al. 2018a, b), they remain largely uncharacterized. Therefore, deciphering the molecular basis for the heat stress tolerance of $P$. haitanensis is important for increasing the commercial value and productivity of red algal species.

The development and application of high-throughput sequencing technology and molecular biology research tools 
have resulted in some recent progress regarding the elucidation of the molecular mechanisms behind $P$. haitanensis heat stress tolerance. For example, Wang et al. (2018b) compared the transcriptomes between Z-61 and wild-type $P$. haitanensis strains, revealing that Z-61 is more thermotolerant than the wild type because of an increase in the transcriptional regulation of genes mainly related to photosynthesis, antioxidant systems, and energy metabolism. In another investigation, 151 heat stress-responsive $P$. haitanensis proteins associated with various processes, mainly including photosynthesis, protein synthesis and degradation, defense response, and energy and carbohydrate metabolism, were identified based on the iTRAQ technique (Shi et al. 2017). On the basis of a lipidomics analysis, Chen et al. (2016b) detected a number of lipid biomarkers for high-temperature stress. Additionally, some candidate genes related to high-temperature responses have been cloned, including genes encoding manganese superoxide dismutase (Yang et al. 2013) and carbonic anhydrases (Chen et al. 2016a). In order to cope with HTs and other abiotic stresses, Pyropia species initiate various defense mechanisms to carry out the appropriate physiological and biochemical changes (Qian et al. 2015; Im et al. 2015; Wang et al. 2018a, 2019, 2020; Zheng et al. 2020). Notably, regardless of the pathway that is activated by HT stress, non-native proteins aggregate and partially unfolded proteins are produced and accumulate in Pyropia species, with detrimental effects on the thalli cells.

Heat shock proteins (HSPs) are molecular chaperones that can prevent irreversible protein aggregations (Liberek et al. 2008; Tyedmers et al. 2010). Based on their molecular weight, homology and functions, HSPs can be classified into several families. Small heat shock proteins (sHSPs) are the most ubiquitous HSPs subgroup, with molecular weights ranging from 12 to $42 \mathrm{kDa}$ (Zhang et al. 2016). They are usually undetectable in vegetative tissues under normal growth conditions but can be induced by environmental stresses and developmental stimulation. In cells exposed to abiotic stresses, sHSPs function as the first line of defense by binding and stabilizing proteins at intermediate stages of folding, assembly, degradation, and translocation across membranes (Waters 2012). Compared to sHSP family in plants, those in red macroalgae seem to be less diverse. To date, only a few sHSPs have been identified in Chondrus crispus (Collén et al. 2013), Porphyra umbilicalis (Brawley et al. 2017), Pyropia yezoensis (Uji et al. 2019), and P. haitanensis (Chen et al. 2015). Identifying and clarifying the physiological functions of sHSPs in response to HT will provide a basis for molecular analyses and the selective breeding of Pyropia species. We previously cloned the $P$. haitanensis Hsp22 gene and found its expression was upregulated more than 1000-folds under HT stress (Chen et al. 2015). The stable genetic transformation system of hygromycin resistance gene by codon optimization has only been reported in P. yezoensis (Uji et al. 2014). Although successful genetic transformation system in $P$. yezoensis has been reported, transgenic strains of $P$. yezoensis can only be propagated through monospore germination, which is a very convenient and fast way of propagation. However, $P$. haitanensis has no such asexual reproduction, resulting in it being difficult to obtain stable transformed cells for $P$. haitanensis. Therefore, sHSP functions remain largely unknown in these species.

Unlike Pyropia species, Chlamydomonas reinhardtii is a unicellular green alga broadly used for elucidating fundamental biological processes. There are several genetic resources and analytical tools available for $C$. reinhardtii, including nuclear, chloroplast, mitochondrial transformation systems, RNAi knockdown methods, a sequenced genome, and hundreds of genetically characterized mutants (Kindle 1990; Shimogawara et al. 1998; Neupert et al. 2012). Moreover, many heterologous genes have been expressed in C. reinhardtii, including genes from microalgae (Cordero et al. 2011), macroalgae (Kim et al. 2011), higher plants (Siripornadulsil et al. 2002), and humans (Rasala and Mayfield 2011). Specifically, Jin et al. (2017) inserted the Pyropia tenera HSP19.3 gene into C. reinhardtii and confirmed that the physiological function of the encoded protein may influence HT tolerance. Accordingly, C. reinhardtii may be suitable for expressing $P$. haitanensis genes. However, the molecular mechanism underlying the HSPs function related to C. reinhardtii HT tolerance is unclear. The objectives of the present study were to establish a stable genetic transformation system of $P$. haitanensis gene (PhHsp22) in C. reinhardtii and investigate the $P h H s p 22$ expression pattern. Additionally, the regulation and expression of genes in response to HT stress were examined via the genome-wide high-throughput transcriptomic analysis of $C$. reinhardtii overexpressing PhHsp22.

\section{Materials and methods}

\section{Algae and growth conditions}

The Pyropia haitanensis Z-61 strain used in this study is tolerant to HT and produces a high yield. It was selected and purified by researchers in the Laboratory of Germplasm Improvements and Applications of Pyropia in Jimei University, Fujian Province, China. Five randomly selected blades ( $15 \pm 2 \mathrm{~cm}$ long) were cultured at $21^{\circ} \mathrm{C}$ with a 10 -h light:14-h dark cycle $\left(50 \mu \mathrm{mol}\right.$ photons $\mathrm{m}^{-2} \mathrm{~s}^{-1}$ irradiance). Additionally, Chlamydomonas reinhardtii strain "CC-400 cw15 mt+" was used to examine the physiological function of PhHsp22. The C. reinhardtii cells were grown in Trisacetate-phosphate (TAP) medium at $25{ }^{\circ} \mathrm{C}$ with shaking (100 rpm) and a 14:10 (light/dark) cycle with cool fluorescent light $\left(50 \mu \mathrm{mol}\right.$ photons $\left.\mathrm{m}^{-2} \mathrm{~s}^{-1}\right)$. 
Sequence and phylogenetic tree analyses of $\mathrm{PhHsp22}$

The PhHsp22 nucleotide sequence, deduced amino acid sequence, and open reading frame were analyzed with tools available on the National Center for Biotechnology Information website (http://www.ncbi.nlm.nih.gov) (Chen et al. 2015). Conserved domains were identified with SMART (http://smart.embl-heidelberg.de/). MEGA6 program was used to construct a phylogenetic tree according to the maximum likelihood method.

\section{RNA extraction and CDNA synthesis}

Total RNA was extracted from $P$. haitanensis and C. reinhardtii and purified with the E.Z.N.A. Plant RNA Kit (Omega, Germany). The quality and quantity of the purified RNA were determined by measuring the absorbance at 260 $\mathrm{nm} / 280 \mathrm{~nm}\left(A_{260} / A_{280}\right)$ and $260 \mathrm{~nm} / 230 \mathrm{~nm}\left(A_{260} / A_{230}\right)$ with a NaroDrop ND-1000 spectrophotometer (LabTech, USA). The integrity of the purified RNA was assessed by $1.2 \%$ agarose gel electrophoresis. The total RNA $(1 \mu \mathrm{g})$ was used as the template for the first-strand cDNA synthesis with the PrimeScript RT reagent kit (TaKaRa, Japan) and random primers. The cDNA samples were diluted with nuclease-free water to $5 \mathrm{ng} \mu \mathrm{L}^{-1}$ and then used as the templates for the quantitative real-time (qRT)-PCR analysis.

\section{Isolation of $\mathrm{PhHsp22}$ and construction of a transformation vector}

The complete PhHsp22 cDNA was cloned with PhHsp22 specific primers (Table S1). The pMD19-T (TaKaRa, Japan) and pChlamy_3 (Invitrogen, USA) vectors were used for constructing the transformation vector. The amplified PhHsp22 sequence was first inserted into the pMD19-T vector. The resulting recombinant plasmid and pChlamy_3 were digested with KpnI and PstI (TaKaRa, Japan), after which the PhHsp22 and linearized pChlamy_3 vectors were ligated to form the transformation vector.

\section{Chlamydomonas reinhardtii nuclear transformation}

The nuclear transformation of $C$. reinhardtii was completed according to a modified version of the electroporation method described by Yamano et al. (2013). Briefly, C. reinhardtii cells were cultured until the cell density reached $1-2 \times 10^{6}$ cells $\mathrm{mL}^{-1}$, after which a $10 \mathrm{~mL}$ aliquot of the cultured cells was centrifuged at $600 \times g$ for $5 \mathrm{~min}$. The pelleted cells were re-suspended in $200 \mu \mathrm{L}$ TAP medium containing $40 \mathrm{mM}$ sucrose for a final density of $1 \times 10^{8}$ cells $\mathrm{mL}^{-1}$. The pChlamyPhHsp22 plasmids $(2 \mu \mathrm{g})$ were linearized with PstI and added to the cell suspension. The cell suspension was added to an electroporation cuvette (ECM830, USA). Parameters were optimized as three Pps of $300 \mathrm{~V}$ with $6 \mathrm{~ms}$ pulse length, $50 \mathrm{~ms}$ pulse interval. After electroporation, the cell suspension from the cuvette was transferred into $10 \mathrm{~mL}$ TAP medium containing $40 \mathrm{mM}$ sucrose and incubated at dim light (1 2 $\mu \mathrm{mol}$ photons $\left.\mathrm{m}^{-2} \mathrm{~s}^{-1}\right)$ for $24 \mathrm{~h}$. Transformed colonies were selected on TAP agar medium containing $10 \mu \mathrm{g} \mathrm{mL}^{-1}$ hygromycin after 7-14 days of growth.

\section{Stress treatment and quantitative real-time PCR analysis}

Chlamydomonas was treated at $33{ }^{\circ} \mathrm{C}$ for 3,6 , and $12 \mathrm{~h}$ to observe the biomass. Cell growth was monitored based on the cell counts determined by measuring the optical density at $750 \mathrm{~nm}$ (Kumar et al. 2013). To assess the effects of HT on gene expression, cDNA was prepared for HT treatment samples for a qRT-PCR analysis with $P h H s p 22$-specific primers (Hsp22QF and Hsp22QF). A $\beta$-tubulin gene was used as an internal control. The primers for qRT-PCR are listed in Table S1. The qRT-PCR was performed with 96-well plates and the ABI 7300 real-time PCR Detection system. The $20 \mu \mathrm{L}$ reaction volumes comprised $10 \mu \mathrm{L} 2 \times$ SYBR Green Master Mix (Takara, China), $0.4 \mu \mathrm{L}$ sense and antisense primers (20 $\mathrm{mM}), 2 \mu \mathrm{L}$ diluted template, $0.4 \mu \mathrm{L}$ ROX Reference Dye $(50 \times)$, and $6.8 \mu \mathrm{L}$ RNA-free water. The qRT-PCR program was as follows: $95^{\circ} \mathrm{C}$ for $30 \mathrm{~s}$ and 40 cycles of $95^{\circ} \mathrm{C}$ for $5 \mathrm{~s}$ and $60{ }^{\circ} \mathrm{C}$ for $31 \mathrm{~s}$. A dissociation curve analysis of the amplification was completed after the PCR cycles to confirm that only one PCR product was amplified and detected.

\section{Transcriptome assembly, annotation, and differential gene expression analysis}

Only RNA samples with an $A_{260} / A_{280}$ ratio of 1.9-2.1 and an $A_{260} / A_{230}>2.0$ were used in the subsequent analyses. The de novo transcriptome assembly and annotation were completed by GENE DENOVO Biotech, Ltd. (Guangzhou, China). Gene expression levels were calculated based on the RPKM (reads per kilobase per million reads) method (Mortazavi et al. 2008). The gene expression data were then used to analyze differentially expressed genes (DEGs) with the DESeq2 software (Love et al. 2014).

\section{Statistical analysis}

All experiments were repeated three times. The standard deviation of the mean was used to determine the precision of the mean. The significance of any differences between the treated and control samples was assessed by a one-way ANOVA and post hoc testing with the LSD test of SPSS 12.0 (SPSS Inc, USA). The threshold for significance was set at $p<0.05$. 


\section{Results}

\section{Identification of PhHsp22 cDNA}

In a previous work, we cloned $P$. haitanensis Hsp22 from a cDNA library based on high-throughput sequencing. PhHsp22 encodes a $19.1 \mathrm{kDa}$ polypeptide with 172 amino acids ( $\mathrm{pI}$ of 5.24) (Chen et al. 2015). The prediction of domain structures in PhHsp22 by SMART revealed the presence of PhHsp22 contained A-crystal structure domain from amino acids 52 to 149 , as well as the HSP20 domain at amino acids 60-164 (Fig. 1a). A phylogenetic analysis supported the existence of a sister-group relationship between $P$. haitanensis and Chondrus crispus but implied that Pyropia species diverged from Cyanophyta, Chlorophyta, Phaeophyta, and land plant species (Fig. 1b).

\section{Confirmation of $C$. reinhardtii transformation and PhHsp22 expression under high-temperature stress}

To investigate whether the constitutive expression of PhHsp22 can improve the HT tolerance of transgenic $C$. reinhardtii, we transformed $C$. reinhardtii with the PhHsp22 coding sequence under the control of a tandem promoter comprising the $H s p 70 A$ and $R b c S 2$ promoters (Fig. $\mathrm{S} 1)$. Many independently regenerated hygromycin-resistant lines were obtained. The transformation was then confirmed by PCR analyses using PhHsp22-specific primers (Table S1). The expected amplicons were produced for all randomly selected hygromycin-resistant lines, but not for the wild-type control, indicating PhHsp22 was integrated into the genome of the hygromycin-resistant lines (Fig. 2a). The 5 th transgenic lines (T5) were selected for the subsequent HT tolerance analyses at the transcriptional and morphological levels. The PhHSP22 expression levels in T5 were determined in a qRT-PCR assay. As shown in Fig. 2c, at least a fifteenfold increase of PhHsp22 expression in transgenic C. reinhardtii was induced by HT stress, with peak expression levels at 33 ${ }^{\circ} \mathrm{C}$ (Fig. 2b). Thus, we evaluated the effects of this temperature on the transgenic $C$. reinhardtii over time (Fig. 2c). As shown in Fig. 2c, the maximal expression of the PhHsp22 gene is detected after $3 \mathrm{~h}$ of induction at $33{ }^{\circ} \mathrm{C}$. The PhHsp 22 expression level decreased gradually after $6 \mathrm{~h}$ and would gradually decline to the normal level after $12 \mathrm{~h}$.

\section{Tolerance of transgenic $C$. reinhardtii to high- temperature stress}

The transgenic $C$. reinhardtii was clearly more tolerant to HT treatment $\left(33{ }^{\circ} \mathrm{C}\right)$ than the WT control (Fig. 3). The cultures for the T5 were obviously greener than WT cultures at $33{ }^{\circ} \mathrm{C}$. The differences between the WT and transgenic cultures exhibited a time-dependent profile.
Fig. 1 Domain and phylogenetic analyses of PhHsp22. a Protein domain analysis. b Phylogenetic tree comprising PhHsp22 and genes encoding small heat shock proteins in other species. The tree was constructed based on amino acid sequences, and the number at each node indicates the percentage of bootstrapping after 1000 replications

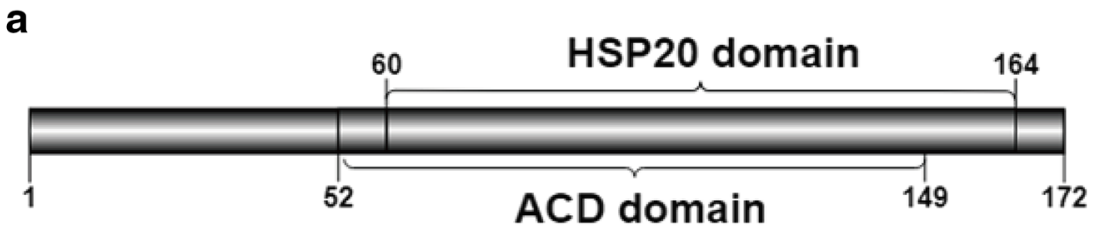

b

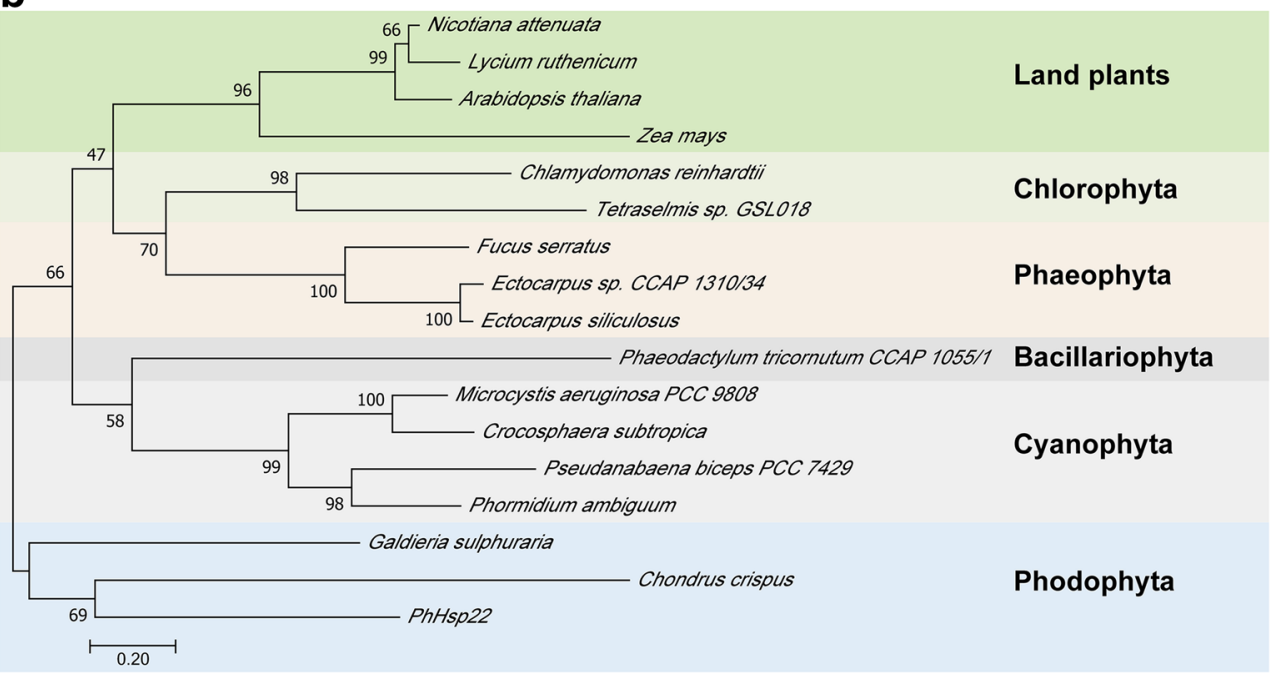


Fig. 2 Transgenic verification of PhHsp22. a Confirmation that the putative transformants carried PhHsp 22 based on PCR analyses with gene-specific primers. b Expression of PhHsp22 under different temperatures. c Expression of $\mathrm{PhHsp} 22$ at $33^{\circ} \mathrm{C}$ over time

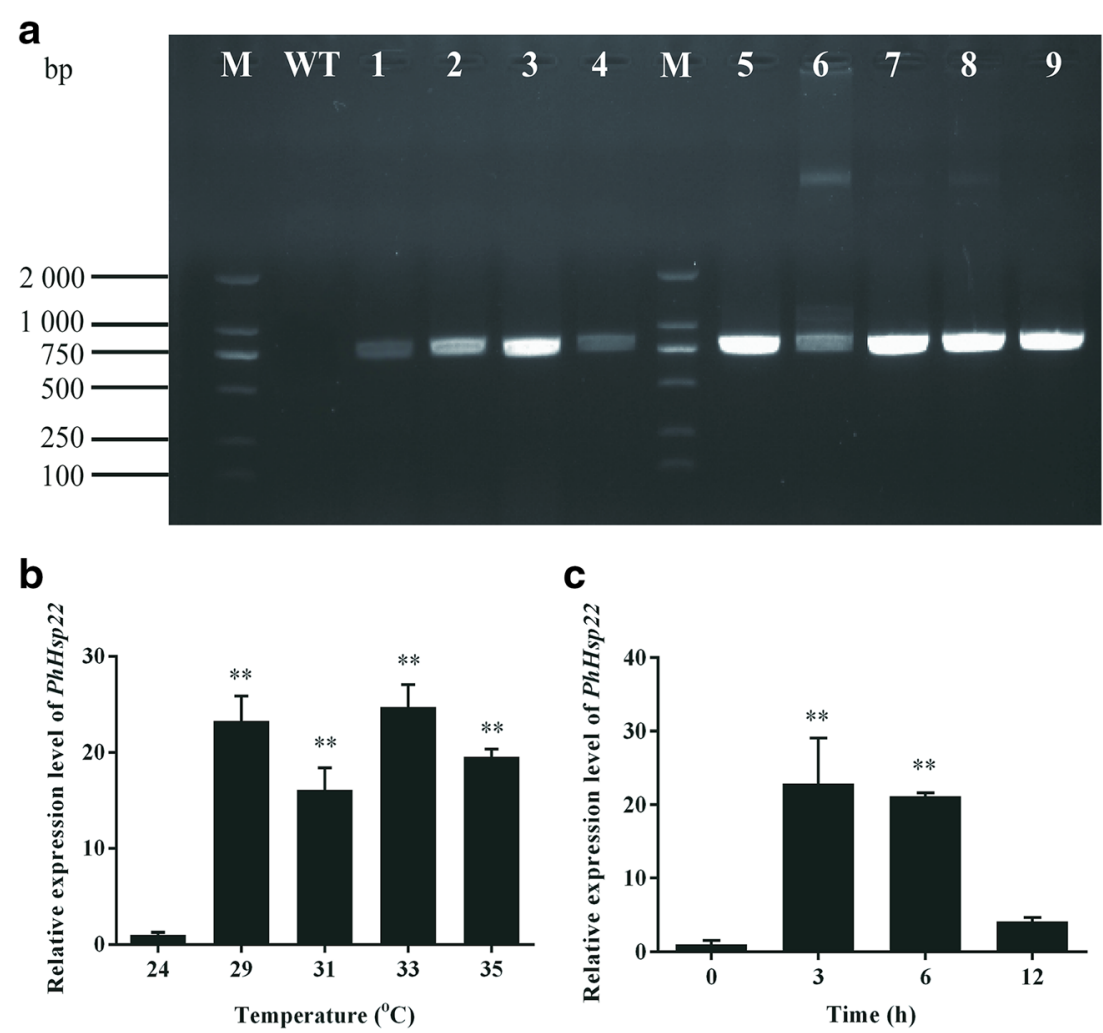

\section{Verification of differentially expressed genes}

To further verify the reliability of the RNA-seq data, 8 unigene sequences were randomly selected from the DEGs of the transcriptome for qPCR analysis. There was a good correlation between the transcript abundances based on the qRT-PCR analysis and the expression profiles determined by RNA-Seq (Figure S2).

\section{Functional analyses of differentially expressed genes}

Based on the abovementioned results, we selected the PhHsp22-overexpressing transgenic C. reinhardtii incubated at $33{ }^{\circ} \mathrm{C}$ for $3 \mathrm{~h}$ for a transcriptome analysis, with the WT $C$. reinhardtii used as the control. The mRNA samples, including different strains of $C$. reinhardtii, were subjected to paired-end sequencing using the Illumina HiSeq2500 platform. Details regarding the transcriptome assembly are provided in supplementary table $\mathrm{S} 2$. The high-temperature stress affected the WT control more than the transgenic C. reinhardtii. Specifically, compared with the WTunstressed (CK) expression levels, the 3-h heat treatment of the WT C. reinhardtii (WT-3 h) upregulated and downregulated the expression of 3666 and 3487 genes, respectively. In contrast, the $3-\mathrm{h}$ heat treatment of the transgenic $C$. reinhardtii (HSP-3 h) upregulated and downregulated the expression of 363 and 556 genes, respectively, relative to the HSP-CK expression levels. Additionally, there were 6958 DEGs between WT-CK and HSP-CK, whereas only 89 DEGs were detected between WT-3 h and HSP-3 h (Fig. 4a). Furthermore, the top
Fig. 3 Effects of PhHsp22 on transgenic Chlamydomonas reinhardtii. a Biomass of C. reinhardtii under heat stress for 3 days. b Concentration of C. reinhardtii under hightemperature stress

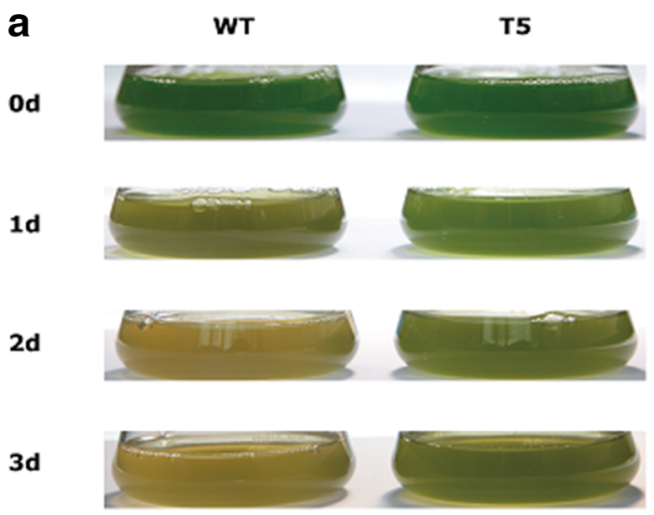


a

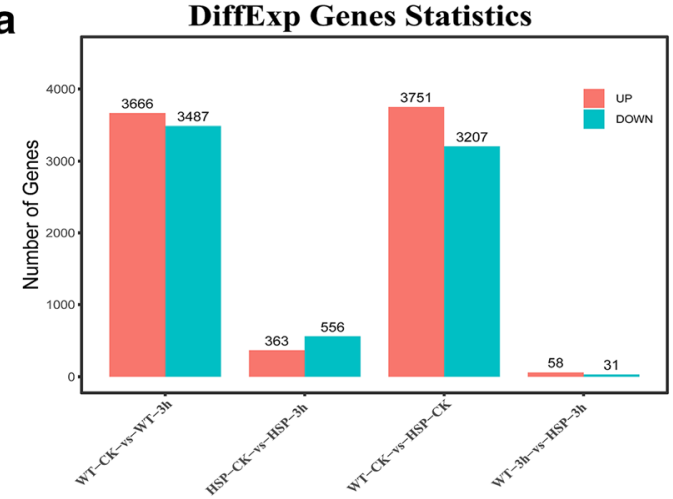

C

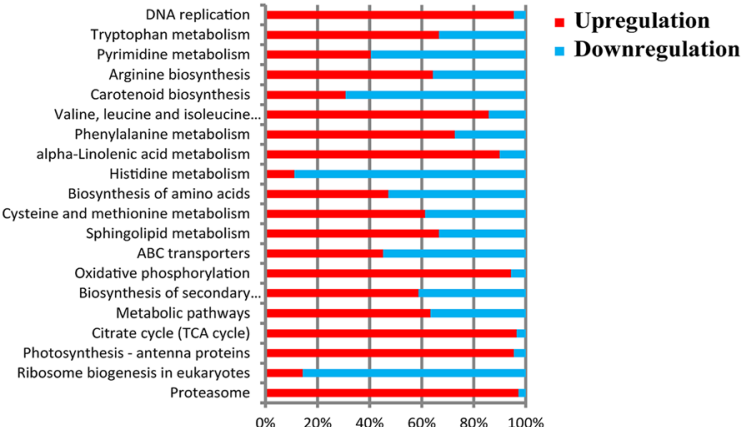

Fig. 4 Functional analyses of differentially expressed genes. a Global overview of the differentially expressed genes (DEGs) among samples (WT-CK, wild-type unstressed control; WT-3 h, wild type exposed to high-temperature stress for $3 \mathrm{~h}$; HSP-CK, transgenic C. reinhardtii

20 enriched KEGG pathways among the DEGs between WT$\mathrm{CK}$ and HSP-CK included proteasome, photosynthesisantenna proteins, citrate cycle, metabolic pathway, and $\mathrm{ABC}$ transporters (Fig. 4b). The expression levels of most of the DEGs related to these pathways were significantly upregulated in HSP-CK (Figs. 4c and 5).

To reveal the functional relationships among the DEGs involved in proteasome, ubiquitin-mediated proteolysis, protein processing in the endoplasmic reticulum, and citrate cycle, a protein-protein interaction network was generated with the STRING program. This network showed that the subgenes encoded diverse proteins, including protein disulfide isomerase (prtp), heat shock protein 70 (HSP70), 20S proteasome alpha subunit E (PAE1), and malate dehydrogenase (MMDH1) (Fig. 6). Moreover, the expression levels of the DEGs associated with the top 20 enriched KEGG pathways were mainly downregulated and upregulated in HSP-3 $\mathrm{h}$ and CK-3 h samples, respectively (Fig. 7).

\section{Discussion}

In the present study we determined that PhHsp22 contained the theoretical domain of ACD and HSP20, which is consistent with the domains of Arabidopsis thaliana sHSPs (Scharf b Top 20 of KEGG Enrichment

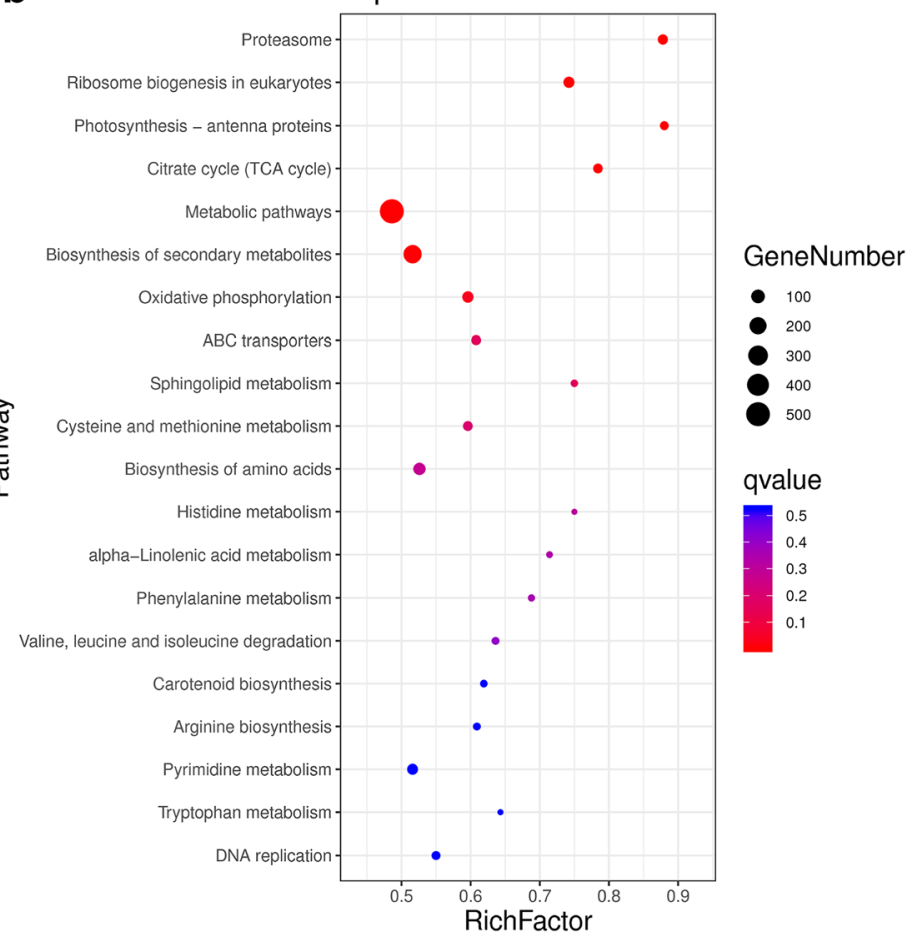

unstressed control; HSP-3 h, transgenic C. reinhardtii exposed to hightemperature stress for $3 \mathrm{~h}$ ). b Top 20 enriched KEGG pathways among the DEGs between WT-CK and HSP-CK. c Number of up- and downregulation genes related to the top 20 enriched KEGG pathways

et al. 2001). The N-terminal amino acid sequence of sHSPs is poorly conserved in other species, indicating that the Nterminal of PhHsp22 has lower conservation (Chen et al. 2015). In contrast, the C-terminal is highly conserved and contains ACD (Scharf et al. 2001). A previous study confirmed that the C-terminal region of PsHsp20 in Porphyra species is highly conserved (Park et al. 2012). Our findings suggest that the PhHsp22 may play a similar function of response to stress at the C-terminal. sHSPs have a high capacity to bind to non-native proteins, but not able to refold, which maybe interact through hydrophobic. Phylogenetic analysis demonstrated that PhHsp22 was poorly clustered with the sHSPs genes from other species (Fig. 1b), which reflect the diversity in the evolution of these genes.

HT stress has a complex effect on cell functions and can completely inactivate enzymes, resulting in protein denaturation (Kampinga et al. 1995). Accordingly, HT may disrupt the synthesis of native proteins in plants, while accelerating the production of HSPs and heat shock factors (Nover et al. 2001; Wang et al. 2004). Our earlier research involving transcriptome analyses and cloning revealed that the synthesis of sHSPs in Pyropia species might increase to mitigate the harmful effects of environmental stresses, including high temperatures (Chen et al. 2015), drought (Shi et al. 2019), and salinity (Wang et al. 2019). Because only P. yezoensis has a stable 


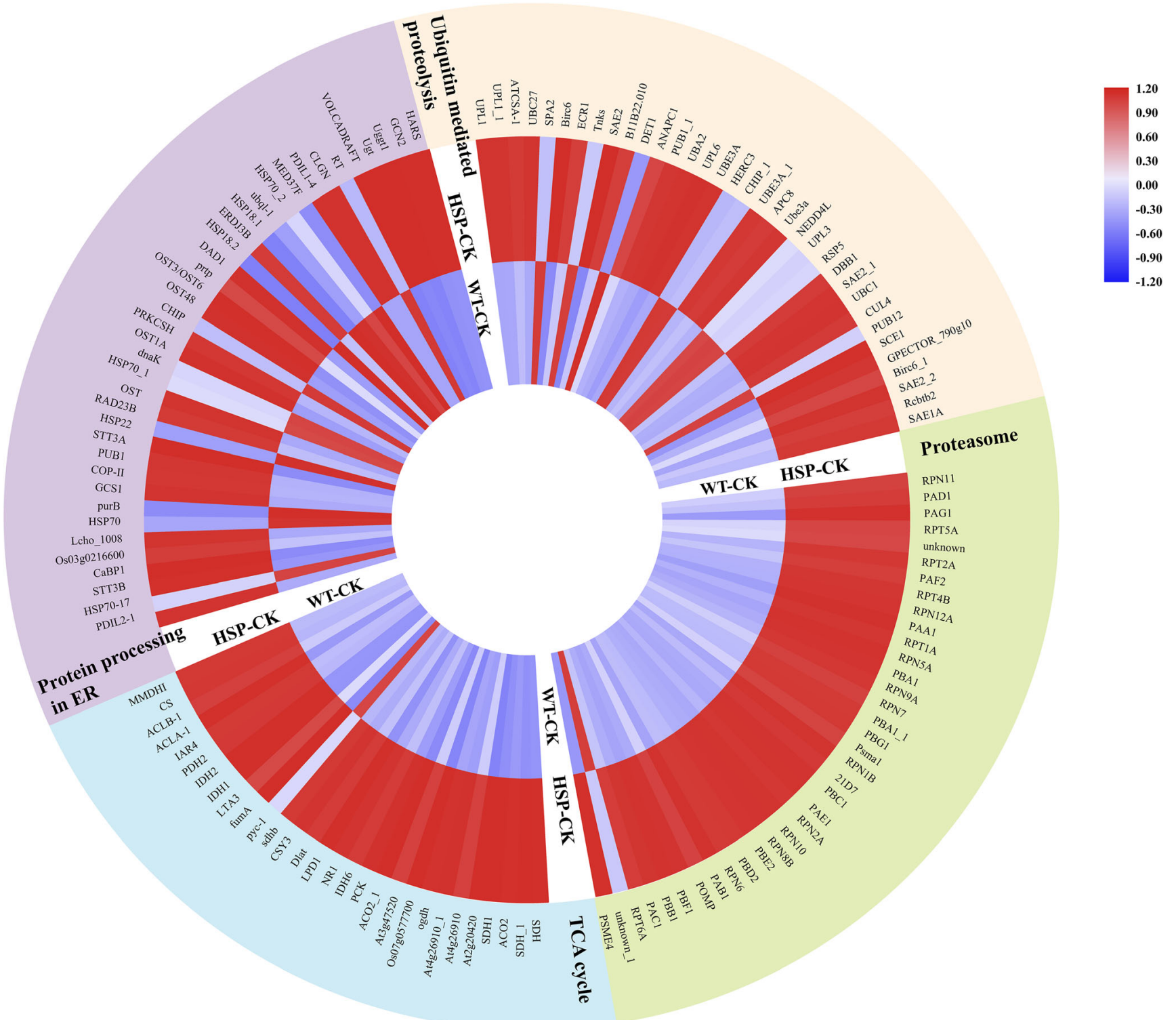

Fig. 5 Heat map of the differentially expressed genes involved in the proteasome, ubiquitin-mediated proteolysis, protein processing in the endoplasmic reticulum (ER), and citrate cycle (TCA cycle) between
WT-CK (wild-type unstressed control) and HSP-CK (transgenic C. reinhardtii unstressed control) transformation system, there is a lack of relevant research on P. haitanensis (Uji et al. 2014). We introduced PhHsp22 into the $C$. reinhardtii genome and analyzed its heat-induced expression in the current study. Driven by the Hsp $70 A$ and RbcS2 tandem promoter, PhHsp22 in transgenic lines could respond positively to heat stress (Fig. 2b). The selection of an appropriate promoter is crucial for gene transformations and transgenes expression in plants (Potenza et al. 2004). Unfortunately, only a few promoters have been confirmed as suitable for red algae (Huddy et al. 2012; Son et al. 2012). There are some promoters in $P$. yezoensis that can be used normally, but they have not been proven to be suitable for P. haitanensis (Fukuda et al. 2008; Uji et al. 2010). The observed upregulated expression of PhHsp22 suggests that $H s p 70 A$ and $R b c S 2$ promoters may be effective for driving transgene expression in C. reinhardtii. In addition, the present result findings imply that PhHsp22 contributes to HT stress response in C. reinhardtii. During the high-temperature (33 ${ }^{\circ} \mathrm{C}$ ) treatment, the promoter of hsp70a-rbcs2 induced high expression of phhsp 22 in transgenic C. reinhardtii from 3 to $6 \mathrm{~h}$ (Fig. 2c). Meanwhile, overexpression of PhHsp 22 contributes to heat stress tolerance in Chlamydomonas (Fig. 3). These results are similar to those of an earlier transgenic study (Jin et al. 2017). The expression of the Pyropia tenera HSP 19.3 gene inserted into the $C$. reinhardtii genome is reportedly upregulated soon after initiating a HT treatment and confers 


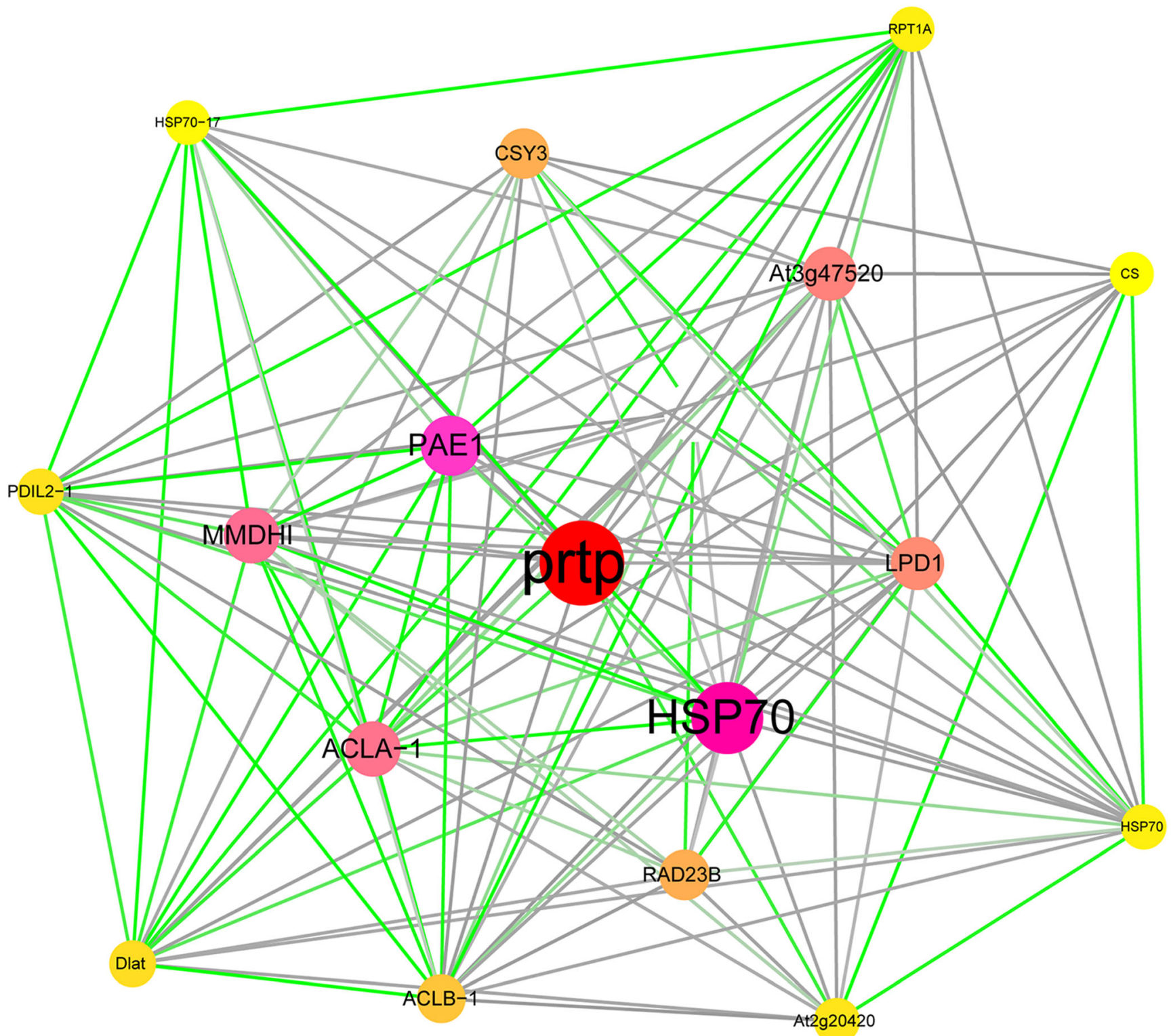

Fig. 6 Protein-protein interaction network for differentially expressed genes related to proteasome, protein processing in endoplasmic reticulum, ubiquitin-mediated proteolysis, and citrate cycle (TCA cycle) in transgenic $C$. reinhardtii based on a STRING analysis. The proteins encoded by the sub-genes in the network are presented in different colors.

the tolerance to heat (Jin et al. 2017). Our data suggest that PhHsp22 from $P$. haitanensis might play a significant role in resisting HT stress.

As chaperones, sHSPs can bind to denatured proteins in an ATP-independent pattern to prevent them from aggregating thereby maintaining cellular homeostasis. PhHsp22 is the most heat stress-responsive $P$. haitanensis sHSPs gene. Moreover, its positive effects on the HT tolerance of transformed $C$. reinhardtii have been confirmed. However, it remains unclear how much of this HT tolerance is due to this gene. Therefore, we compared the transcriptional profiles of WT and PhHsp22-overexpressing $C$. reinhardtii exposed to
Red color represents a degree between 634 and 425, whereas purple color represents a degree between 425 and 200 . The green line indicates a combined score greater than 543 , whereas the gray line indicates a combined score of less than 543 . The circle size corresponds to the number of protein interactions. Protein annotations are listed in Table S2

the same HT stress. The results suggest that PhHsp22 mainly affects the proteasome, photosynthesis-antenna proteins, citrate cycle, oxidative phosphorylation, biosynthesis of amino acid, protein processing in endoplasmic reticulum, ubiquitinmediated proteolysis, and other pathways (Figs. 4 and 5). Thus, the transgenic $C$. reinhardtii had fewer DEGs than WT control following the same HT treatment (Fig. 4a), indicating that PhHsp22 can maintain the cellular homeostasis of C. reinhardtii in response to HT stress.

Misfolded and denatured proteins inevitably accumulate in Pyropia thalli under HT stress conditions. The misfolded and unfolded proteins are perceived as endoplasmic reticulum 
Fig. 7 Numbers of up- and downregulated genes among the top 20 enriched KEGG pathways between HSP-CK and HSP-3 h (a) as well as between WT-CK and WT- 3 h. b WT-CK, wildtype unstressed control; WT-3 h, wild type exposed to heat stress for $3 \mathrm{~h}$; HSP-CK, transgenic C. reinhardtii unstressed control; HSP-3 h, transgenic C. reinhardtii exposed to heat stress for $3 \mathrm{~h}$ a

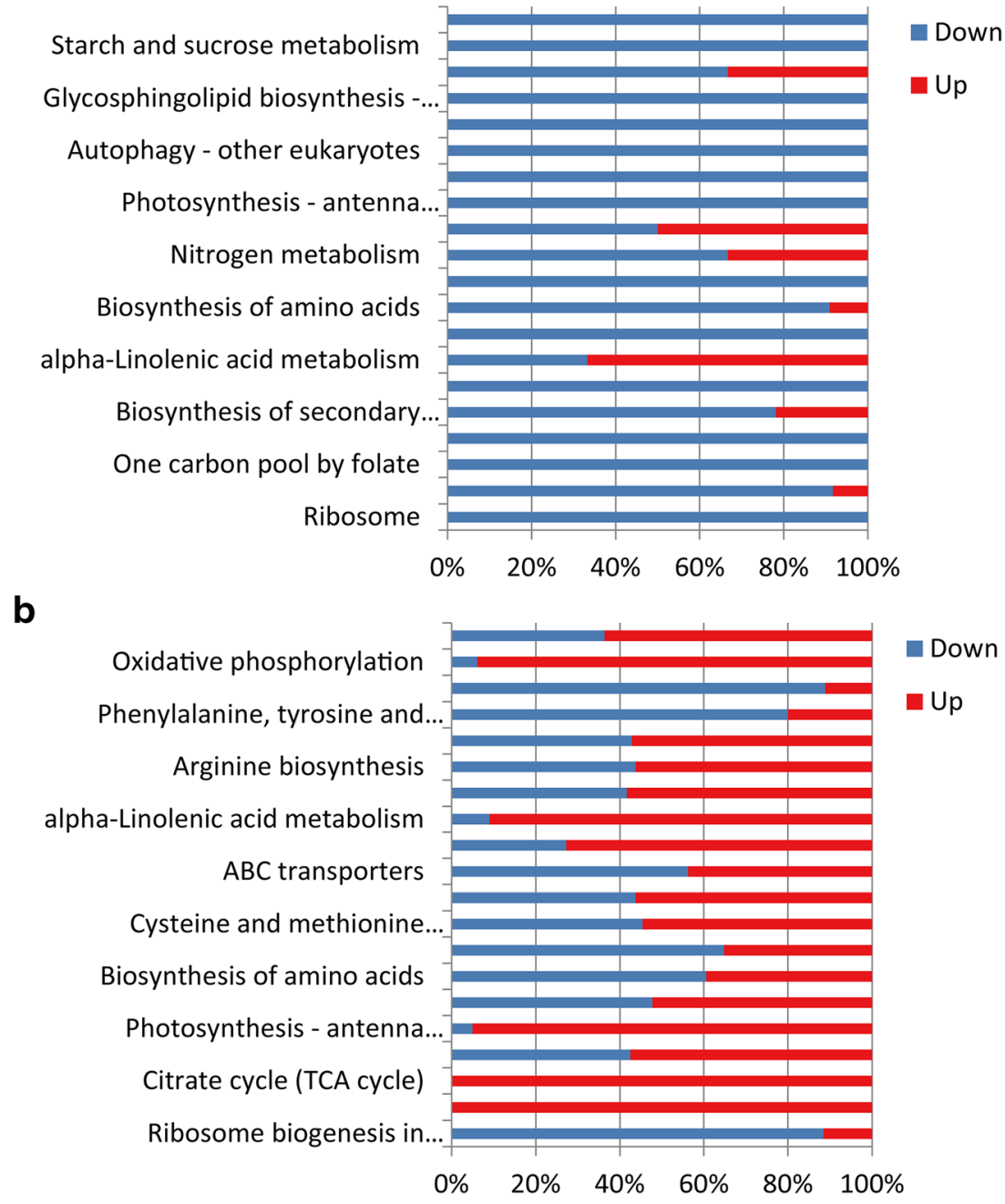

stress by specific sensor proteins in the endoplasmicreticulum membrane, ultimately resulting in the expression of genes encoding chaperones. In addition, misfolded and unfolded proteins are ubiquitinized by $26 \mathrm{~S}$ proteasome for endoplasmic reticulum-associated degradation (ERAD) or inhibit protein translation to reduce the abundance of synthetic proteins and avoid endoplasmic reticulum stress (Helenius and Aebi 2004; Zhu 2016). The quality control system recognizes proteins in the endoplasmic reticulum based on interactions with calnexin and calreticulin (Helenius and Aebi 2004). Correctly folded proteins are transported to the Golgi apparatus through COP II, whereas misfolded and denaturation proteins are degraded by a proteasome or refolded via HSPs and other molecular chaperones (Wang et al. 2018a; Shi et al. 2019; Wen et al. 2020). In a recent study, all of the genes annotated by ubiquitin-mediated proteolysis, including those encoding the ubiquitin-activating enzyme, ubiquitin-conjugating enzyme, ubiquitin ligase, and most of the genes related to $26 \mathrm{~S}$ proteasome pathway exhibited upregulated in $P$. haitanensis under severe HT conditions (Wang et al. 2018a). This HT-induced expression may be due to the fact that the ubiquitin- proteasome system mediates the removal of damaged and/or misfolded proteins and modulates regulatory protein contents. Additionally, Wen et al. (2020) pointed out that increases in the abundance of HSPs and proteins involved in ubiquitinmediated proteasome activity stabilize the dynamic balance between protein folding and removal in $P$. haitanensis in response to hypersalinity. Here, the expression levels of 26 of 35 DEGs related to ubiquitin-mediated proteolysis as well as 25 of 38 DEGs related to protein processing in endoplasmic reticulum were upregulated in the PhHsp22-overexpressing transgenic C. reinhardtii (Fig. 5). Furthermore, PhHsp22 upregulated the expression of all but one of the 36 DEGs related to proteasomes which are large multi-subunit proteases in the cytosol (Bochtler et al. 1999). Proteasomes can recognize, unfold, and digest protein substrates that have been marked for degradation with an ubiquitin moiety (Bochtler et al. 1999). Thus, the $C$. reinhardtii cells expressing PhHsp22 may be protected from fluctuations in environmental conditions via buffering proteasome and HSPs pools (Hanna et al. 2007). HSP expansions mediating protein homeostasis might reflect the adaptation of Pyropia species to the intertidal 
environment (Wang et al. 2019, 2020). The protein-protein interaction network analysis also indicated that HSPs influence key regulatory activities (Fig. 6). Therefore, our results imply that PhHsp22 contributes to the HT tolerance of C. reinhardtii by maintaining cellular homeostasis.

Additionally, we previously determined that severe HT stress substantially upregulates the expression of genes related to encoding energy metabolism in $P$. haitanensis, thereby enhancing carbohydrate metabolism to provide energy and the related carbon skeletons for key metabolic processes (Wang et al. 2018a) such as the citrate cycle is responsible for the oxidation of respiratory substrates that drive ATP synthesis (Sweetlove et al. 2010) and considerably influences the ability of plants to resist the effects of abiotic stresses (Mailloux et al. 2007). First, pyruvate dehydrogenase converts pyruvic acid to acetyl coenzyme A (acetyl-CoA). The formation of citric acid from acetylCoA and oxaloacetic acid via a reaction catalyzed by citric acid synthetase represents the beginning of the citrate cycle. Citric acid synthetase is the rate-limiting enzyme of the citrate cycle. We observed that compared with the WT levels, the expression levels of 28 of 29 DEGs related to pyruvate dehydrogenase, malate dehydrogenase, succinate dehydrogenase, citrate synthase, and other key enzymes were obviously upregulated in the transgenic $C$. reinhardtii cells (Fig. 5). This is consistent with previous studies, $C$. reinhardtii switching from regular metabolism to a specialized metabolism for stress protection (Schroda et al. 2015). Within the first $3 \mathrm{~h}$ of HS stress, several of TCA/ glycolytic enzymes and the glyoxylate cycle were significantly downregulated, while the main metabolism metabolites are rapidly depleted (Hemme et al. 2014). At the same time, the quantitative results also verify this point (Figure S2). Therefore, PhHsp22 may mediate energy metabolism in P. haitanensis exposed to high-temperature stress. The enhancement of the citrate cycle and other energy metabolism-related processes not only increase energy production but also provide sufficient amounts of carbon skeletons for amino acid biosynthesis, DNA replication, arginine biosynthesis, and protein processing (Fig. 4c) to protect thalli from HT stress.

\section{Conclusion}

In this study, the function of the PhHsp22 gene from $P$. haitanensis was verified by transformed into C. reinhardtii. The early upregulation of $P h H s p 22$ expression following a HT treatment suggests this gene contributes to the early response to HT stress. Moreover, its HT-induced expression increases the survival of transgenic $C$. reinhardtii via its effects on cellular energy, secondary metabolites, HSPs, and proteasome pools. The results of this investigation may clarify the regulatory effects of HSPs on the HT tolerance of P. haitanensis. Furthermore, PhHsp 22 may be useful for the breeding of new macroalgal strains adapted to global warming.

Supplementary Information The online version contains supplementary material available at https://doi.org/10.1007/s10811-020-02351-6.

Acknowledgments We thank the Liwen Bianji, Edanz Group China (www.liwenbianji.cn/ac), for editing the English text of a draft of this manuscript.

Authors' contributions C.T.X., W.L.W., and J.Z.S. conceived and designed the experiment. J.C., J.Z.S., and W.L.W. performed experiments and data analysis. C.T.X. and C.S.C. contributed by planning, supervising, and financing the work. D.H.J. and Y.X. helped to prepare the materials and reagents. J.C., J.Z.S., and W.L.W. drafted and revised the manuscript. All authors read and approved the final manuscript.

Funding This work was supported by the "National Natural Science Foundation of China (grant number: 31872567 and 41806185), National Key R\&D Program of China (Grant No: 2018YFD0900106 and 2018YFD0901500) and Fujian Province Science and Technology Major Project (2019NZ08003)" and supported by "China Agriculture Research System (Grant No: CARS-50).”

\section{Compliance with ethical standards}

Conflict of interest The authors declare that they have no conflicts of interest.

Open Access This article is licensed under a Creative Commons Attribution 4.0 International License, which permits use, sharing, adaptation, distribution and reproduction in any medium or format, as long as you give appropriate credit to the original author(s) and the source, provide a link to the Creative Commons licence, and indicate if changes were made. The images or other third party material in this article are included in the article's Creative Commons licence, unless indicated otherwise in a credit line to the material. If material is not included in the article's Creative Commons licence and your intended use is not permitted by statutory regulation or exceeds the permitted use, you will need to obtain permission directly from the copyright holder. To view a copy of this licence, visit http://creativecommons.org/licenses/by/4.0/.

\section{References}

Blouin NA, Brodie JA, Grossman AC, Xu P, Brawley SH (2011) Porphyra: a marine crop shaped by stress. Trends Plant Sci 16:2937

Bochtler M, Ditzel L, Groll M, Hartmann C, Huber R (1999) The proteasome. Annu Rev Biophys Biomol Struct 28:295-317

Brawley SH, Blouin NA, Ficko-Blean E, Wheeler GL, Lohr M, Goodson HV, Jenkins JW, Blaby-Haas CE, Helliwell KE, Chan CX, Marriage TN, Bhattacharya D, Klein AS, Badis Y, Brodie J, Cao Y, Collén J, Dittami SM, Gachon CMM, Green BR, Karpowicz SJ, Kim JW, Kudahl UJ, Lin S, Michel G, Mittag M, Olson BJSC, Pangilinan JL, Peng Y, Qiu H, Shu S, Singer JT, Smith AG, Sprecher BN, Wagner V, Wang W, Wang ZY, Yan J, Yarish C, Zäuner-Riek S, Zhuang Y, Zou Y, Lindquist EA, Grimwood J, Barry KW, Rokhsar DS, Schmutz J, Stiller JW, Grossman AR, Prochnik SE (2017) Insights into the red algae and eukaryotic 
evolution from the genome of Porphyra umbilicalis (Bangiophyceae, Rhodophyta). Proc Natl Acad Sci U S A 114: E6361-E6370

Cao M, Xu KP, Yu XZ, Bi GQ, Liu Y, Kong FN, Sun PP, Tang XH, Du GY, Ge Y, Wang DM, Mao YX (2020) A chromosome level genome assembly of Pyropia haitanensis (Bangiales, Rhodophyta). Mol Ecol Resour 20:216-227

Chen CS, Ji DH, Xie CT, Xu Y, Liang Y, Zheng YJ, Shi XZ, Wang FX, Zhao ML (2008) Preliminary study on selecting the HT resistance strains and economic traits of Porphyra haitanensis (in Chinese). Acta Oceanol Sin 30:100-106

Chen YT, Xu Y, Ji DH, Chen CS, Xie CT (2015) Cloning and expression analysis of two small heat shock protein (sHsp) genes from Pyropia haitanensis (in Chinese). J Fish China 39:182-192

Chen CS, Dai ZZ, Xu Y, Ji DH, Xie CT (2016a) Cloning, expression, and characterization of carbonic anhydrase genes from Pyropia haitanensis (Bangiales, Rhodophyta). J Appl Phycol 28:1403-1417

Chen J, Li M, Yang R, Luo Q, Xu J, Ye Y, Yan X (2016b) Profiling lipidome changes of Pyropia haitanensis in short-term response to high-temperature stress. J Appl Phycol 28:1903-1913

Collén J, Porcel B, Carré W et al (2013) Genome structure and metabolic features in the red seaweed Chondrus crispus shed light on evolution of the Archaeplastida. Proc Natl Acad Sci U S A 110:52475252

Cordero BF, Couso I, León R, Rodríguez H, Vargas MÁ (2011) Enhancement of carotenoids biosynthesis in Chlamydomonas reinhardtii by nuclear transformation using a phytoene synthase gene isolated from Chlorella zofingiensis. Appl Microbiol Biotechnol 91:341-351

Fukuda S, Mikami K, Uji T, Park EJ, Ohba T, Asada K, Kitade Y, Endo H, Kato I, Saga N (2008) Factors influencing efficiency of transient gene expression in the red macrophyte Porphyra yezoensis. Plant Sci 174:329-339

Hanna J, Meides A, Zhang DP, Finley D (2007) A ubiquitin stress response induces altered proteasome composition. Cell 129:747-759

Helenius A, Aebi M (2004) Roles of N-linked glycans in the endoplasmic reticulum. Annu Rev Biochem 73:1019-1049

Hemme D, Veyel D, Muhlhaus T, Sommer F, Jüppner J, Unger AK, Sandmann M, Fehrle I, Schönfelder S, Steup M, Geimer S, Kopka J, Giavalisco P, Schroda M (2014) Systems-wide analysis of acclimation responses to long-term heat stress and recovery in the photosynthetic model organism Chlamydomonas reinhardtii. Plant Cell 26:4270-4297

Huddy SM, Meyers AE, Coyne VE (2012) Transformation of lacZ using different promoters in the commercially important red alga, Gracilaria gracilis. Afr J Biotechnol 11:1879-1885

Hwang EK, Yotsukura N, Pang SJ, Su L, Shan TF (2019) Seaweed breeding programs and progress in eastern Asian countries. Phycologia 58:484-495

Im S, Choi S, Hwang MS, Park EJ, Jeong WJ, Choi DW (2015) De novo assembly of transcriptome from the gametophyte of the marine red algae Pyropia seriata and identification of abiotic stress response genes. J Appl Phycol 27:1343-1353

Jin Y, Yang S, Im S, Jeong WJ, Park E, Choi DW (2017) Overexpression of the small heat shock protein, PtsHSP19.3 from marine red algae, Pyropia tenera (Bangiales, Rhodophyta) enhances abiotic stress tolerance in Chlamydomonas. J Plant Biotechnol 44:287-295

Kampinga HH, Brunsting JF, Stege GJ, Burgman PW, Konings AW (1995) Thermal protein denaturation and protein aggregation in cells made thermotolerant by various chemicals: role of heat shock proteins. Exp Cell Res 219:536-546

Kim E, Park HS, Jung YJ, Choi DW, Jeong WJ, Park HS, Hwang MS, Park EJ, Gong YG (2011) Identification of the high-temperature response genes from Porphyra seriata (Rhodophyta) ESTs and enhancement of heat tolerance of Chlamydomonas (Chlorophyta) by expression of the Porphyra HTR2 gene. J Phycol 47:821-828
Kindle KL (1990) High-frequency nuclear transformation of Chlamydomonas reinhardtii. Proc Natl Acad Sci U S A 87:12281232

Kumar A, Falcao VR, Sayre RT (2013) Evaluating nuclear transgene expression systems in Chlamydomonas reinhardtii. Algal Res 2: 321-332

Liberek K, Lewandowska A, Ziętkiewicz S (2008) Chaperones in control of protein disaggregation. EMBO J 27:328-335

Love MI, Huber W, Anders S (2014) Moderated estimation of fold change and dispersion for RNA-seq data with DESeq2. Genome Biol 15:550

Mailloux RJ, BeÂ Riault R, Lemire J, Singh R, CheÂnier DR, Hamel RD, Appanna VD (2007) The tricarboxylic acid cycle, an ancient metabolic network with a novel twist. PLoS One 2:e690

Mortazavi A, Williams BA, McCue K, Schaeffer L, Wold B (2008) Mapping and quantifying mammalian transcriptomes by RNASeq. Nat Methods 5:621-628

Neupert J, Shao N, Lu Y, Bock R (2012) Genetic transformation of the model green alga Chlamydomonas reinhardtii. In: Dunwell J, Wetten A (eds) Transgenic Plants. Methods in Molecular Biology (Methods and Protocols), vol 847. Humana Press, NY, pp 35-47

Nover L, Bharti K, Döring P, Mishra SK, Ganguli A, Scharf KD (2001) Arabidopsis and the heat stress transcription factor world: how many heat stress transcription factors do we need? Cell Stress \& Chaperones 6:177-189

Park HS, Jeong WJ, Kim EC, Jung Y, Lim JM, Hwang MS, Park EJ, Ha DS, Choi DW (2012) Heat shock protein gene family of the Porphyra seriata and enhancement of heat stress tolerance by PsHSP70 in Chlamydomonas. Mar Biotechnol 14:332-342

Potenza C, Aleman L, Sengupta-Gopalan C (2004) Targeting transgene expression in research, agricultural, and environmental applications: promoters used in plant transformation. Vitro Cell Dev Biol - Plant 40:1-22

Qian F, Luo Q, Yang R, Zhu Z, Chen H, Yan X (2015) The littoral red alga Pyropia haitanensis uses rapid accumulation of floridoside as the desiccation acclimation strategy. J Appl Phycol 27:621-632

Rasala BA, Mayfield SP (2011) The microalga Chlamydomonas reinhardtii as a platform for the production of human protein therapeutics. Bioeng Bugs 2:50-54

Sahoo D, Tang X, Yarish C (2002) Porphyra-the economic seaweed as a new experimental system. Curr Sci 83:1313-1316

Scharf KD, Siddique M, Vierling E (2001) The expanding family of Arabidopsis thaliana small heat stress proteins and a new family of proteins containing $\alpha$-crystallin domains (Acd proteins). Cell Stress Chaperones 6:225-237

Schroda M, Hemme D, Mühlhaus T (2015) The Chlamydomonas heat stress response. The Plant Journal 82:466-480

Shi JZ, Chen YT, Xu Y, Ji DH, Chen CS, Xie CT (2017) Differential proteomic analysis by iTRAQ reveals the mechanism of Pyropia haitanensis responding to HT stress. Sci Rep 7:44734

Shi JZ, Wang WL, Lin YH, Xu K, Xu Y, Ji DH, Chen CS, Xie CT (2019) Insight into transketolase of Pyropia haitanensis under desiccation stress based on integrative analysis of omics and transformation. BMC Plant Biol 19:475

Shimogawara K, Fujiwara S, Grossman A, Usuda H (1998) Highefficiency transformation of Chlamydomonas reinhardtii by electroporation. Genetics 148:1821-1828

Siripornadulsil S, Traina S, Verma DPS, Sayre RT (2002) Molecular mechanisms of proline-mediated tolerance to toxic heavy metals in transgenic microalgae. The Plant Cell 14:2837-2847

Son SH, Ahn JW, Uji T, Choi DW, Park EJ, Hwang MS, Liu JR, Choi D, Mikami K, Jeong WJ (2012) Development of an expression system using the heat shock protein 70 promoter in the red macroalga, Porphyra tenera. J Appl Phycol 24:79-87 
Sweetlove LJ, Beard KF, Nunes-Nesi A, Fernie AR, Ratcliffe RG (2010) Not just a circle: flux modes in the plant TCA cycle. Trends Plant Sci 15:462-470

Tyedmers J, Mogk A, Bukau B (2010) Cellular strategies for controlling protein aggregation. Nat Rev Mol Cell Biol 11:777-788

Uji T, Takahashi M, Saga N, Mikami K (2010) Visualization of nuclear localization of transcription factors with cyan and green fluorescent proteins in the red alga Porphyra yezoensis. Mar Biotechnol 12: $150-159$

Uji T, Hirata R, Fukuda S, Mizuta H, Saga N (2014) codon-optimized bacterial antibiotic gene used as selection marker for stable nuclear transformation in the marine red alga Pyropia yezoensis. Mar Biotechnol 16:251-255

Uji T, Gondaira Y, Fukuda S, Mizuta H, Saga N (2019) Characterization and expression profiles of small heat shock proteins in the marine red alga Pyropia yezoensis. Cell Stress Chaperones 24:223-233

Wang W, Vinocur B, Shoseyov O, Altman A (2004) Role of plant heatshock proteins and molecular chaperones in the abiotic stress response. Trends Plant Sci 9:244-252

Wang WL, Teng F, Lin YH, Ji DH, Xu Y, Chen CS, Xie CT (2018a) Transcriptomic study to understand thermal adaptation in a HTtolerant strain of Pyropia haitanensis. PLoS One 13:e0195842

Wang WL, Lin YH, Teng F, Ji DH, Xu Y, Chen CS, Xie CT (2018b) Comparative transcriptome analysis between heat-tolerant and sensitive Pyropia haitanensis strains in response to HT stress. Algal Res 29:104-112

Wang WL, Xu Y, Chen TX, Xing L, Xu K, Ji DH, Chen CS, Xie CT (2019) Regulatory mechanisms underlying the maintenance of homeostasis in Pyropia haitanensis under hypersaline stress conditions. Sci Total Environ 662:168-179
Wang WL, Chen TX, Xing L, Xu Y, Xu K, Ji DH, Chen CS, Xie CT (2020) Investigating the mechanisms underlying the hyposaline tolerance of intertidal seaweed, Pyropia haitanensis. Algal Res 47: 101886

Waters ER (2012) The evolution, function, structure, and expression of the plant sHSPs. J Exp Bot 64:391-403

Wen J, Wang WL, Xu K, Ji DH, Xu Y, Chen CS, Xie CT (2020) Comparative analysis of proteins involved in energy metabolism and protein processing in Pyropia haitanensis at different salinity levels. Front Plant Sci 7:415

Yamano T, Iguchi H, Fukuzawa H (2013) Rapid transformation of Chlamydomonas reinhardtii without cell-wall removal. J Biosci Bioeng 115:691-694

Yang R, Liu W, Zhang XL, Shen ML, Sun X, Chen HM (2013) Sequences of Mn-SOD gene from Pyropia haitanensis (Bangiales, Rhodophyta) and its expression under heat shock. Bot Mar 56:249 259

Zhang J, Chen HY, Wang HH, Li B, Yi YJ, Kong FJ, Liu JY, Zhang HX (2016) Constitutive expression of a tomato small heat shock protein gene LeHSP21 improves tolerance to high-temperature stress by enhancing antioxidation capacity in tobacco. Plant Mol Biol Rep 34:399-409

Zheng HY, Wang WL, Xu K, Xu Y, Ji DH, Chen CS, Xie CT (2020) $\mathrm{Ca}^{2+}$ influences heat shock signal transduction in Pyropia haitanensis. Aquaculture 516:734618

Zhu JK (2016) Abiotic stress signaling and responses in plants. Cell 167: 313-324

Publisher's note Springer Nature remains neutral with regard to jurisdictional claims in published maps and institutional affiliations. 